PROCEEDINGS OF THE

AMERICAN MATHEMATICAL SOCIETY

Volume 136, Number 6, June 2008, Pages 2125-2133

S 0002-9939(08)09187-9

Article electronically published on February 21, 2008

\title{
ON MULTIPLIERS FOR HARDY-SOBOLEV SPACES
}

\author{
FRANK BEATROUS AND JACOB BURBEA
}

(Communicated by Mei-Chi Shaw)

\begin{abstract}
It is shown that membership of holomorphic functions in HardySobolev spaces in the unit ball cannot be characterized by finiteness of any integral norm. In addition, sufficient conditions are given for a holomorphic function to be a pointwise multiplier of a Hardy-Sobolev space.
\end{abstract}

\section{INTRODUCTION}

A natural question to ask about a space of holomorphic functions is whether membership of a given holomorphic function in the space can be characterized by finiteness of some integral norm. For example, the Bergman-Sobolev space $A_{1 / 2}^{2}$ consisting of all holomorphic functions on the unit ball $B$ in $\mathbb{C}^{n}$ with square integrable derivatives of order $1 / 2$ can be characterized as the space of holomorphic functions on $B$ having boundary values that are square integrable on the sphere (the classical Hardy space). On the other hand, membership in the BergmanSobolev space $A_{1}^{2}$, consisting of holomorphic functions with square integrable first derivatives, cannot be characterized by finiteness of any integral.

In this paper, we show that membership in any Hardy-Sobolev space $H_{s}^{p}$ cannot be characterized by finiteness of any integral norm (see Theorem 4.3 for the precise statement). This extends a result of Arveson 1 for a particular Hilbert space which may be realized as the Hardy-Sobolev space $H_{(n-1) / 2}^{2}$. As in Arveson's argument, the key technical step is the existence of functions in the ball algebra that are not in Hardy-Sobolev spaces.

A closely related issue is identification of the pointwise multipliers on the spaces under consideration. It was recently shown by Ryan and Stoll [6] that the HardySobolev space $H_{s}^{p}$ forms a Banach algebra when $s p>n$, and therefore is its own multiplier algebra. Characterization of the multipliers on other Hardy-Sobolev spaces remains open, but we give sufficient conditions for a function to be a pointwise multiplier in Section 5. We do not know if our sufficient conditions are also necessary.

We want to thank Joe Cima for drawing our attention to some of the issues addressed here. We also thank Pamela Ryan and Manfred Stoll for providing us with an early draft of their paper 6].

Received by the editors January 26, 2007, and, in revised form, April 2, 2007.

2000 Mathematics Subject Classification. Primary 32A35.

(C)2008 American Mathematical Society Reverts to public domain 28 years from publication 


\section{Preliminaries}

For $0<q<\infty$ and $\lambda \in \mathbb{C}$ with $|\lambda|<1$, let

$$
k_{q}(\lambda)=(1-\lambda)^{-q}=\sum \frac{(q)_{j}}{j !} \lambda^{j}
$$

and for $z$ and $\zeta$ in the unit ball $B$ of $\mathbb{C}^{n}$, let $K_{q}(z, \zeta)=k_{q}(\langle z, \zeta\rangle)$. In other words,

$$
K_{q}(z, \zeta)=\frac{1}{(1-\langle z, \zeta\rangle)^{q}}=\sum \frac{(q)_{|\alpha|}}{\alpha !} z^{\alpha} \bar{\zeta}^{\alpha}
$$

where

$$
(q)_{k}=q(q+1) \cdots(q+k-1)=\frac{\Gamma(q+k)}{\Gamma(q)} .
$$

In the sum on the right of (2.1), $\alpha$ runs over all multi-indices in $\mathbb{Z}_{+}^{n}$, and $|\alpha|=\sum \alpha_{j}$. When $q$ is $n$ or $n+1, K_{q}$ is the classical Szegö or Bergman kernel, respectively, In general $K_{q}$ is a positive definite sesquiholomorphic kernel on $B$, which is the reproducing kernel of a uniquely determined functional Hilbert space of holomorphic functions on $B$, which we denote by $\mathcal{H}_{q}$. To be explicit, $\mathcal{H}_{q}$ consists of all holomorphic functions

$$
f(z)=\sum a_{\alpha} z^{\alpha}
$$

on $B$ such that

$$
\|f\|_{q}^{2}=\sum \frac{\alpha !}{(q)_{|\alpha|}}\left|a_{\alpha}\right|^{2}<\infty .
$$

The associated inner product

$$
\left\langle\sum a_{\alpha} z^{\alpha}, \sum b_{\alpha} z^{\alpha}\right\rangle_{q}=\sum \frac{\alpha !}{(q)_{|\alpha|}} a_{\alpha} \bar{b}_{\alpha}
$$

satisfies

$$
f(z)=\langle f, K(\cdot, z)\rangle_{q}
$$

for every $f \in \mathcal{H}_{q}$ and every $z \in B$. The monomials $z^{\alpha}$ form a complete orthogonal system for $\mathcal{H}_{q}$, and

$$
\left\|z^{\alpha}\right\|_{q}^{2}=\frac{\alpha !}{(q)_{|\alpha|}}
$$

It will also be convenient to define

$$
K_{0}(z, \zeta)=-\frac{1}{\langle z, \zeta\rangle} \log (1-\langle z, \zeta\rangle)=\sum \frac{1}{j+1}\langle z, \zeta\rangle^{j}
$$

where $\log$ denotes the principal branch of the logarithm. As before, $K_{0}$ is positive definite on $B \times B$ and is the reproducing kernel of a functional Hilbert space $\mathcal{H}_{0}$ consisting of all holomorphic functions $f(z)=\sum a_{\alpha} z^{\alpha}$ on $B$ satisfying

$$
\|f\|_{0}^{2}=\sum \frac{\alpha !(|\alpha|+1)}{|\alpha| !}\left|a_{\alpha}\right|^{2}<\infty
$$

The Hilbert space $\mathcal{H}_{n}$ is the classical Hardy space $H^{2}(B)$, and

$$
\|f\|_{n}^{2}=\sup _{0<r<1} \int_{S}|f(r z)|^{2} d \sigma(z)
$$


where $d \sigma$ is the normalized surface measure on the unit sphere $S$. For $q>n, \mathcal{H}_{q}$ is a weighted Bergman space:

$$
\|f\|_{q}^{2}=\frac{\Gamma(q)}{\pi^{n} \Gamma(q-n)} \int_{B}|f(z)|^{2}\left(1-|z|^{2}\right)^{q-n-1} d v(z),
$$

where $d v$ is the usual Euclidean volume element on $\mathbb{C}^{n}$. For these matters, see [5, Chapters 3 and 7 .

For $0 \leq q<n, \mathcal{H}_{q}$ may be viewed as a Hardy-Sobolev space. For $0<p<\infty$ and $s \in \mathbb{R}$, we define the Hardy-Sobolev space $H_{s}^{p}(B)$ to be the space of holomorphic functions on $B$ satisfying

$$
\|f\|_{p, s}=\left\|D^{s} f\right\|_{H^{p}}
$$

where

$$
D=1+\sum z_{j} \frac{\partial}{\partial z_{j}}
$$

with fractional powers defined in terms of power series:

$$
D^{s}\left(\sum a_{\alpha} z^{\alpha}\right)=\sum(1+|\alpha|)^{s} a_{\alpha} z^{\alpha} .
$$

With this notation, $\mathcal{H}_{q}=H_{(n-q) / 2}^{2}$ with equivalent, but not equal, norms. For a detailed exposition of these matters, see [3].

\section{A FUnCtion IN THE BALl ALGEBRA}

Since the spaces $\mathcal{H}_{q}$ may be realized as Hardy or weighted Bergman spaces when $q \geq n$, it is clear that $\mathcal{H}_{q}$ contains the ball algebra when $q \geq n$. In this section we will use the homogeneous polynomials of Ryll and Wojtaszczyk [7] to construct a function in the ball algebra that is not in any of the Hilbert spaces $\mathcal{H}_{q}$ with $0 \leq q<n$.

In [7] it is shown that there is a sequence $\left(p_{k}\right)$ of homogeneous polynomials in the unit sphere of $H^{\infty}(B)$ such that $p_{k}$ has degree $k$ and

$$
\left\|p_{k}\right\|_{n}=\left\|p_{k}\right\|_{H^{2}} \geq \frac{\sqrt{\pi}}{2^{n}} .
$$

Since $p_{k}$ is homogeneous of degree $k$, it follows from (2.2) and Stirling's Formula that

$$
\left\|p_{k}\right\|_{q}^{2}=\frac{(n)_{k}}{(q)_{k}}\left\|p_{k}\right\|_{n}^{2} \geq \frac{(n)_{k}}{(q)_{k}} \frac{\pi}{4^{n}} \geq c k^{n-q}
$$

for a positive constant $c$ depending only on $n$ and $q$. Let

$$
\varphi=\sum_{j=1}^{\infty} \frac{1}{j^{2}} p_{2^{j}} .
$$

Since $\left\|p_{k}\right\|_{\infty}=1$, the series converges uniformly on $\bar{B}$ to a function $\varphi$ in the ball algebra. Moreover, since the polynomials $p_{k}$ are homogeneous of different degrees, they are orthogonal in $\mathcal{H}_{q}$, so

$$
\|\varphi\|_{q}^{2}=\sum \frac{\left\|p_{2^{j}}\right\|_{q}^{2}}{j^{4}} \geq c \sum \frac{2^{j(n-q)}}{j^{4}}
$$

The sum on the right diverges if $q<n$, so $\varphi \notin \mathcal{H}_{q}$ for $q<n$. We have proved

Theorem 3.1. The function $\varphi$ defined by (3.1) is in the ball algebra, but is not in $\mathcal{H}_{q}$ for $0<q<n$. 
For clarity, we have restricted our attention to the Hilbert spaces $\mathcal{H}_{q}$, but essentially the same construction can be used to show that some other unitarily invariant Hilbert spaces of holomorphic functions do not contain the ball algebra. To be precise, for any sequence $\left(c_{j}\right)$ of nonnegative numbers such that $\sum \frac{c_{j}}{j !} \lambda^{j}$ converges on the unit disk, define

$$
K(z, \zeta)=\sum \frac{c_{j}}{j !}\langle z, \zeta\rangle^{j}
$$

Then $K$ is the reproducing kernel for a unitarily invariant functional Hilbert space $\mathcal{H}_{K}$ of holomorphic functions on the ball. The result is as follows.

Theorem 3.2. Suppose there is a summable sequence $\left(\varepsilon_{j}\right)$ of positive numbers and an increasing sequence $\left(k_{j}\right)$ of positive integers such that $\sum \varepsilon_{j}^{2} \frac{(n)_{k_{j}}}{c_{k_{j}}}$ diverges. Then the function $\varphi=\sum \varepsilon_{j} p_{k_{j}}$ is in the ball algebra, but is not in $\mathcal{H}_{K}$.

Proof. As before, the series $\sum \varepsilon_{j} p_{k_{j}}$ converges uniformly on $\bar{B}$ and its sum $\varphi$ is therefore in the ball algebra. Also as before, the monomials $p_{k}$ are pairwise orthogonal in $\mathcal{H}_{K}$, and

SO

$$
\left\|p_{k}\right\|_{K}^{2}=\frac{(n)_{k}}{c_{k}}\left\|p_{k}\right\|_{n}^{2}
$$

$$
\begin{aligned}
\|\varphi\|_{K}^{2} & =\sum_{j} \varepsilon_{j}^{2}\left\|p_{k_{j}}\right\|_{K}^{2} \\
& =\sum_{j} \varepsilon_{j}^{2} \frac{(n)_{k_{j}}}{c_{k_{j}}}\left\|p_{k_{j}}\right\|_{n}^{2} \\
& \geq c \sum_{j} \varepsilon_{j}^{2} \frac{(n)_{k_{j}}}{c_{k_{j}}} \\
& =\infty
\end{aligned}
$$

and therefore $\varphi \notin \mathcal{H}_{K}$.

\section{HARDY-SoboleV SPACES}

In this section, we show that Hardy-Sobolev spaces do not contain certain holomorphic Lipschitz functions. We begin with a refinement of Theorem 3.1 .

Theorem 4.1. There is a function in the ball algebra that is not in $H_{s}^{p}$ for any $1 \leq p \leq \infty$ and $s>0$.

Proof. Let $\varphi$ be the function defined by (3.1). Since the spaces $H_{s}^{p}$ are monotonically decreasing in $p$, it suffices to show that $D^{s} \varphi \notin H^{1}$ for any $s>0$. Let

$$
f=D^{s} \varphi=\sum \frac{\left(1+2^{j}\right)^{s}}{j^{2}} p_{2^{j}}
$$

with uniform convergence on compact subsets of $B$. Since the polynomials $p_{k}$ are orthogonal, for any $0<r<1$,

SO

$$
\int_{S} f(r z) \overline{p_{2^{j}}(z)} d \sigma(z)=r^{2^{j}} \frac{\left(1+2^{j}\right)^{s}}{j^{2}}\left\|p_{2^{j}}\right\|_{H^{2}}^{2}
$$

$$
\sup _{0<r<1}\left|\int_{S} f(r z) \overline{p_{2^{j}}(z)} d \sigma(z)\right|=\frac{\left(1+2^{j}\right)^{s}}{j^{2}}\left\|p_{2^{j}}\right\|_{H^{2}}^{2} \geq \frac{\pi}{4^{n}} \frac{\left(1+2^{j}\right)^{s}}{j^{2}}
$$


which tends to $\infty$ as $j \rightarrow \infty$. On the other hand, since $\left\|p_{k}\right\|_{\infty}=1$,

$$
\sup _{0<r<1}\left|\int_{S} f(r z) \overline{p_{2^{j}}(z)} d \sigma(z)\right| \leq \sup _{0<r<1} \int_{S}|f(r z)| d \sigma(z)=\|f\|_{H^{1}}<\infty .
$$

This contradiction completes the proof.

For $s>0$, the holomorphic Lipschitz space $\Lambda_{s}$ is defined to be the space of all holomorphic functions $f$ on $B$ satisfying

$$
\|f\|_{\Lambda_{s}}=\sup \left|D^{k} f(z)\right|\left(1-|z|^{s}\right)^{s-k}<\infty,
$$

where $k$ is the least integer greater than $s$.

Theorem 4.2. Let $0<p<\infty$ and $s>0$. For any $0<\varepsilon<s$ there is a function $\varphi \in \Lambda_{\varepsilon}$ such that $\varphi \notin H_{s}^{p}$.

Proof. If $\Lambda_{\varepsilon} \subset H_{s}^{p}$, then the inclusion must be continuous by the Closed Graph Theorem. Let

$$
q_{k}=(1+k)^{-\varepsilon} p_{k}
$$

where the $p_{k}$ are the Ryll-Wojtaszczyk polynomials. Thus $\left\|D^{\varepsilon} q_{k}\right\|_{\infty}=\left\|p_{k}\right\|_{\infty}=1$, and it follows that

$$
\left\|q_{k}\right\|_{\Lambda_{\varepsilon}} \leq c
$$

for some positive constant $c$ depending on $\varepsilon$ and the dimension $n$. On the other hand, for $0<p \leq 2$,

$$
\begin{aligned}
\left\|q_{k}\right\|_{p, s}^{p} & =\int_{S}\left|D^{s-\varepsilon} p_{k}(z)\right|^{p} d \sigma(z) \\
& =(1+k)^{p(s-\varepsilon)} \int_{S}\left|p_{k}(z)\right|^{p} d \sigma(z) \\
& \geq(1+k)^{p(s-\varepsilon)} \int_{S}\left|p_{k}(z)\right|^{2} d \sigma(z) \\
& \geq(1+k)^{p(s-\varepsilon)} \frac{\pi}{4^{n}} .
\end{aligned}
$$

The first inequality follows from the fact that $p \leq 2$ and $\left|p_{k}\right| \leq 1$ pointwise. Since the right side tends to $\infty$ as $k \rightarrow \infty$, and, by (4.1), the sequence $\left(q_{k}\right)$ is bounded in $\Lambda_{\varepsilon}$, there is no continuous inclusion of $\Lambda_{\varepsilon}$ into $H_{s}^{p}$, which establishes the theorem when $0<p \leq 2$. For $p>2$, it's only necessary to observe that $H_{s}^{p} \subset H_{s}^{2}$, so the result follows from the known case $p=2$.

It is natural to ask which spaces of holomorphic functions can be characterized by an integral norm. It is well known (see [3]) that the Hilbert spaces $\mathcal{H}_{q}$ are weighted Bergman spaces when $q>n$, and that $\mathcal{H}_{n}$ is a Hardy space. It was shown by Arveson [1] that $\mathcal{H}_{1}$ cannot be characterized by an integral norm when $n>1$. Here we show that no Hardy-Sobolev space $H_{s}^{p}$ with $s>0$ can be characterized by an integral norm. In particular, $\mathcal{H} q$ cannot be so characterized for $0 \leq q<n$. This is elementary when $n=1$, but seems to be new in higher dimensions.

To be precise, for any Borel measure $\mu$ on $\bar{B}$ and any increasing function $\lambda$ on $[0, \infty)$ with $\lambda(0)=0$, let $H_{\mu}^{\lambda}$ denote the set of all holomorphic functions $f$ on $B$ satisfying

$$
\Lambda(f)=\limsup _{r \rightarrow 1^{-}} \int_{B} \lambda(|f(r z)|) d \mu(z)<\infty .
$$


Theorem 4.3. Let $0<p \leq \infty$ and $s>0$. Then $H_{s}^{p} \neq H_{\mu}^{\lambda}$ as sets for every $\mu, \phi$.

Proof. Suppose $H_{s}^{p}=H_{\mu}^{\lambda}$. Since the constant function 1 is in $H_{s}^{p}$, we have $\int \lambda(1) d \mu<\infty$, so $\mu$ is finite. But then for any $f$ in the ball algebra and any $0<r<1$, we have

$$
\int_{B} \lambda(|f(r z)|) d \mu(z) \leq \sup |f| \mu(\bar{B}),
$$

so $f \in H_{\mu}^{\lambda}=H_{s}^{p}$, contradicting Theorem 4.2 .

\section{Multipliers}

A functional (quasi-)Banach spac $\rrbracket^{1} \mathcal{B}$ on a set $X$ is a (quasi-)Banach space of complex-valued functions on $X$ such that for every $x \in X$ the functional $f \mapsto f(x)$ is continuous on $\mathcal{B}$. We call a functional quasi-Banach space $\mathcal{B}$ on $X$ total if for each $x \in X$ there is an $f \in \mathcal{B}$ such that $f(x) \neq 0$.

A complex-valued function $\varphi$ on $X$ is a multiplier for $\mathcal{B}$ if the multiplication operator $M_{\varphi} f=\varphi f$ maps $\mathcal{B}$ into itself, in which case it follows from the Closed Graph Theorem that $M_{\varphi}$ is continuous on $\mathcal{B}$. The set $\mathcal{M}(B)$ of multipliers on $\mathcal{B}$ is an algebra of functions on $X$, which, if $\mathcal{B}$ is total, may be viewed as a (quasi-)Banach algebra by defining the norm of a multiplier $\varphi$ to be the operator norm of the multiplication operator $M_{\varphi}$.

Lemma 5.1. Let $\mathcal{B}$ be a total functional quasi-Banach space on $X$. Then every multiplier $\varphi$ for $\mathcal{B}$ is bounded pointwise by its multiplier norm.

Proof. Let $M_{\varphi}^{*}$ be the adjoint of the multiplication operator, which is an operator on the dual space $\mathcal{B}^{*}$. Let $x \in X$ and let $\varepsilon_{x}$ be the evaluation functional at $x$. For any $f \in \mathcal{B}$,

$$
\left(M_{\varphi}^{*} \varepsilon_{x}\right) g=\varepsilon_{x}\left(M_{\varphi} f\right)=\varphi(x) f(x)=\varphi(x) \varepsilon_{x} f
$$

so

$$
M_{\varphi}^{*} \varepsilon_{x}=\varphi(x) \varepsilon_{x} .
$$

Therefore

$$
\left\|M_{\varphi}^{*}\right\|\left\|\varepsilon_{x}\right\| \geq|\varphi(x)|\left\|\varepsilon_{x}\right\|
$$

and since $\varepsilon_{x} \neq 0$ by hypothesis,

$$
|\varphi(x)| \leq\left\|M_{\varphi}^{*}\right\| \leq\left\|M_{\varphi}\right\| .
$$

In the case of Hardy-Sobolev and Bergman spaces, the function space contains the constants, and so any multiplier must itself be a member of the space, and must therefore be holomorphic. Therefore we have

Lemma 5.2. The multiplier algebra of a Hardy-Sobolev space is contained in $H^{\infty}$, and the $H^{\infty}$ norm of a multiplier is less than or equal to its operator norm.

Conversely, it is clear that any $H^{\infty}$ function is a multiplier for any Hardy or Bergman space and that the multiplier norm is less than or equal to the $H^{\infty}$ norm. Therefore

Lemma 5.3. The multiplier algebra for any Hardy or Bergman space is precisely $H^{\infty}$, and the multiplier and $H^{\infty}$ norms are equal.

\footnotetext{
${ }^{1}$ A quasi-norm on a vector space $V$ is a function $v \mapsto\|v\|$ from $V$ to $[0, \infty)$ that vanishes only at the zero vector, is positively homogeneous, and satisfies $\|v+w\| \leq C(\|v\|+\|w\|)$ for some positive constant $C$. A quasi-Banach space is a Cauchy complete quasi-normed vector space.
} 
The above lemma does not extend to any Hardy-Sobolev space $H_{s}^{p}$ with $s>0$. For these spaces, there are bounded functions that are not multipliers. Indeed, by Theorem 4.2 there is a holomorphic Lipschitz function that is not in $H_{s}^{p}$, and therefore not a multiplier for $H_{s}^{p}$. This extends a result Arveson [1], who showed that there is a function in the ball algebra that is not in $H_{(n-1) / 2}^{2}=\mathcal{H}_{1}$ and is therefore not a multiplier for that space. Moreover, there are multipliers on $H_{s}^{p}$ with multiplier norm strictly greater than the $H^{\infty}$ norm.

Theorem 5.4. Let $0<p<\infty$ and let $s>0$. For any $C>0$ there is a multiplier $\varphi$ with $\|\varphi\|_{\infty} \leq 1$ and $\left\|M_{\varphi}\right\| \geq C$.

Proof. Suppose the theorem fails. Then there is a $C$ such that for every multiplier $\varphi$ we have

$$
\left\|M_{\varphi}\right\| \leq C\|\varphi\|_{\infty}
$$

By Theorem 4.2, there is a function $\varphi$ in the ball algebra that is not a multiplier. Let $p_{k}$ be a sequence of polynomials that converges uniformly to $\varphi$. By (5.1), the sequence $M_{p_{k}}$ is a Cauchy sequence in the operator norm, which must then be norm convergent to a continuous operator $T$ on $H_{s}^{p}$. It follows that

$$
(T f)(z)=\lim _{k \rightarrow \infty}\left(M_{p_{k}} f\right)(z)=\lim _{k \rightarrow \infty} p_{k}(z) f(z)=\varphi(z) f(z)
$$

for every $f \in H_{s}^{p}$ and every $z \in B$, and so $M_{\varphi}=T$ is continuous on $H_{s}^{p}$, a contradiction.

In order to identify multipliers on Hardy-Sobolev spaces, we will need an elementary variant of Hölder's Inequality.

Lemma 5.5. Let $0<p, q, r<\infty$ with $\frac{1}{p}=\frac{1}{q}+\frac{1}{r}$. Then for any $f \in H^{r}$ and $g \in H^{q}$ the product $f g$ is in $H^{p}$ and

$$
\|f g\|_{p} \leq\|f\|_{r}\|g\|_{q} .
$$

Proof. Apply Hölder's Inequality to the functions $|f|^{p}$ and $|g|^{p}$.

We next give a sufficient condition for multipliers on $H_{s}^{p}$ when $s$ is small.

Theorem 5.6. Let $0<p<\infty$ and let $s$ be an integer with $0<s<n / p$. Any $\varphi \in H_{s}^{n / s} \cap H^{\infty}$ is a multiplier for $H_{s}^{p}$.

Before proceeding with the proof, we note that the spaces $H_{s}^{n / s}$ are monotonically decreasing in the parameter $s$ :

$$
H_{t}^{n / t} \subset H_{s}^{n / s} \quad \text { for } t \geq s .
$$

Moreover, each of the spaces $H_{s}^{n / s}$ is contained in BMOA, and when $s \geq n$, is contained in the ball algebra. In particular, it follows that intersecting with $H^{\infty}$ is redundant when $s \geq n$. See [3] and [2] for details on these matters.

Proof. Let $f \in H_{s}^{p}$ and let $\gamma$ be a multi-index with $|\gamma| \leq s$. Then

$$
\partial^{\gamma}(\varphi f)=\sum_{\alpha+\beta=\gamma} c_{\alpha, \beta} \partial^{\alpha} \varphi \partial^{\beta} f,
$$

where $c_{\alpha, \beta}$ are positive constants. It therefore suffices to show that

$$
\partial^{\alpha} \varphi \partial^{\beta} f \in H^{p}
$$


for all multi-indices $\alpha$ and $\beta$ with $|\alpha|+|\beta| \leq s$. Let's first dispense with the case $\alpha=0$. By hypothesis, $\varphi \in H^{\infty}$, so

$$
\left\|\varphi \partial^{\beta} f\right\|_{p} \leq\|\varphi\|_{\infty}\left\|\partial^{\beta} f\right\|_{p}<\infty
$$

For $\alpha \neq 0$, we have

$$
\partial^{\alpha} \varphi \in H_{s-|\alpha|}^{n / s} \subset H^{r}
$$

where $r=n /|\alpha|$. The last inclusion is a consequence of the Sobolev Embedding Lemma for Hardy-Sobolev spaces (see [3]). Similarly,

$$
\partial^{\beta} f \in H_{s-|\beta|}^{p} \subset H_{|\alpha|}^{p} \subset H^{q},
$$

where

$$
\frac{n}{q}=\frac{n}{p}-|\alpha|=\frac{n}{p}-\frac{n}{r}
$$

Thus

$$
\frac{1}{p}=\frac{1}{q}+\frac{1}{r}
$$

so it follows from Lemma 5.5 that $\partial^{\alpha} \varphi \partial^{\beta} f \in H^{p}$, which completes the proof.

For $s=n / p$ we have

Theorem 5.7. If $s=n / p$ is an integer, and if $\varphi \in H_{t}^{p}$ for some $t>s$, then $\varphi$ is a multiplier for $H_{s}^{p}$.

Proof. Since $H_{t}^{p} \subset H_{n / p}^{p} \cap H^{\infty}$, the proof can proceed as that of Theorem 5.6, except for the estimation of terms of the form $\left(\partial^{\alpha} \varphi\right) f$ with $|\alpha|=n / p$. Letting $\varepsilon=t-s$, we have

where $\frac{n}{q}=\frac{n}{p}-\varepsilon$ and

$$
\partial^{\alpha} \varphi \in H_{\varepsilon}^{p} \subset H^{q}
$$

$$
f \in H_{n / p}^{p} \subset B M O A \subset \bigcap_{0<r<\infty} H^{r} .
$$

In particular, $f \in H^{r}$ with $r=n / \varepsilon$. Since $\frac{1}{p}=\frac{1}{q}+\frac{1}{r}$, it follows that $\left(\partial^{\alpha} \varphi\right) f \in H^{p}$.

For $s>n / p$, the multipliers for $H_{s}^{p}$ are characterized by a result of Ryan and Stoll [6].

Theorem 5.8 (Ryan and Stoll). Let $s$ be a positive integer, satisfying $s>n / p$, or $s=n / p$ with $p \leq 1$. Then $H_{s}^{p}$ is an algebra, and therefore is its own multiplier algebra.

The above results on multipliers for Hardy-Sobolev spaces extend readily to Bergman-Sobolev spaces. We define $A_{q, s}^{p}$ to be the space of all holomorphic functions $f$ on $B$ satisfying

$$
\|f\|_{p, q, s}=\left(\int_{B}\left|D^{s} f(z)\right|^{p}\left(1-|z|^{2}\right)^{q-1} d v(z)\right)^{1 / p}<\infty .
$$

Here $s \geq 0,0<p<\infty$, and $q>0$. It is known that $A_{q_{1}, s_{1}}^{p}=A_{q_{2}, s_{2}}^{p}$ when $q_{1}-p s_{1}=q_{2}-p s_{2}$.

Theorem 5.9. (1) If $0 \leq s<q / p$, then the multiplier algebra for $A_{q, s}^{p}$ is $H^{\infty}$.

(2) If $\frac{q}{p} \leq s<\frac{n+q}{p}$, then each function in $A_{q, s}^{(n+q) / s} \cap H^{\infty}$ is a multiplier. 
(3) If $s=(n+q) / p$, then the multiplier algebra for $A_{q, s}^{p}$ contains $A_{q, t}^{p}$ for every $t>s$.

(4) If $s>(n+q) / p$ or if $p \leq 1$ and $s=(n+q) / p$, then $A_{q, s}^{p}$ is an algebra, which is therefore its own multiplier algebra.

Proof. Item 1 follows from the fact that $A_{q, s}^{p}$ is the same as the weighted Bergman space $A_{q-p s, 0}^{p}$ when $p s<q$. When the parameter $s$ is an integer, the remaining items can be proved by the same arguments used in the Hardy space case. For noninteger values of $s$, we have $A_{q, s}^{p}=A_{q_{1}, s_{1}}^{p}$ where $s_{1}$ is the least integer greater than or equal to $s$ and $q_{1}=q+p\left(s_{1}-s\right)$, so the result follows from the integer case.

We now return to the Hardy-Sobolev spaces in the case $p=2$. In this case (see [3]),

$$
H_{s}^{2}=A_{q, t}^{2}
$$

whenever $t=s+q / 2$. In the case $p=2$, this observation allows us to remove the restriction that the parameter $s$ is an integer, which was needed previously.

Theorem 5.10. (1) If $0<s<\frac{n}{2}$, then each function in $H_{n / 2}^{2} \cap H^{\infty}$ is a multiplier.

(2) The multiplier algebra for $H_{n / 2}^{2}$ contains $H_{t}^{2}$ for every $t>n / 2$.

(3) If $s>n / 2$, then $H_{s}^{2}$ is an algebra, which is therefore its own multiplier algebra.

Item 1 can be restated as

Corollary 5.11. For $0<q<\infty$ the multiplier algebra for $\mathcal{H}_{q}$ contains $\mathcal{H}_{0} \cap H^{\infty}$.

\section{REFERENCES}

[1] W. Arveson, Subalgebras of $C^{*}$-algebras III: Multivariable operator theory, Acta Math. 181 (1998), 159-228. MR1668582(2000e:47013)

[2] F. Beatrous, Boundary continuity of holomorphic functions in the ball, Proc. Amer. Math. Soc. 97 (1986), 23-29. MR831380 (87d:32008)

[3] F. Beatrous and J. Burbea, Holomorphic Sobolev spaces on the ball, Dissertationes Math. 256 (1989), 1-57. MR 1010151 (90k:32010)

[4] Z. Chen, Characterizations of Arveson's Hardy space, Complex Variables 48 (2003), 453-465. MR1974382(2004c:32010)

[5] W. Rudin, Function Theory in the Unit Ball of $\mathbb{C}^{n}$, Springer-Verlag, 1980. MR601594 (82i:32002)

[6] P. Ryan and M. Stoll, Hardy-Sobolev Spaces and Banach Algebras on the Unit Ball in $\mathbb{C}^{n}$, preprint.

[7] J. Ryll and P. Wojtaszczyk, On homogeneous polynomials on a complex ball, Trans. Amer. Math. Soc. 276 (1983), 107-116. MR684495 (84f:32004) 15260

Department of Mathematics, University of Pittsburgh, Pittsburgh, Pennsylvania

E-mail address: beatrous@pitt.edu

Department of Mathematics, University of Pittsburgh, Pittsburgh, Pennsylvania 15260

E-mail address: burbea@pitt.edu 